\title{
Efficacy of psychodynamic short-term psychotherapy for depressed breast cancer patients: study protocol for a randomized controlled trial
}

\author{
Rüdiger Zwerenz ${ }^{1 * \dagger}$, Manfred E Beutel ${ }^{1 \dagger}$, Barbara H Imruck', Jörg Wiltink', Antje Haselbacher ${ }^{1}$, Christian Ruckes $^{2}$, \\ Heinz Schmidberger ${ }^{3}$, Gerald Hoffmann ${ }^{4}$, Marcus Schmidt ${ }^{5}$, Uwe Köhler ${ }^{6}$, Dagmar Langanke ${ }^{7}$, \\ Rolf-Dieter Kortmann ${ }^{8}$, Susanne Kuhnt ${ }^{9}$, Gregor Weißflog ${ }^{9}$, Yvette Barthel $^{9}$, Katja Leuteritz ${ }^{9}$ and Elmar Brähler ${ }^{9}$
}

\begin{abstract}
Background: There is a lack of psychotherapeutic trials of treatments of comorbid depression in cancer patients. Our study determines the efficacy of a manualized short-term psychodynamic psychotherapy and predictors of outcome by personality and quality of the therapeutic relationship.

Methods/design: Eligible breast cancer patients with comorbid depression are assigned to short-term psychodynamic psychotherapy (up to $20+5$ sessions) or to treatment as usual (augmented by recommendation for counseling center and physician information). We plan to recruit a total of 180 patients (90 per arm) in two centers. Assessments are conducted pretreatment, after 6 (treatment termination) and 12 months (follow-up). The primary outcome measures are reduction of the depression score in the Hospital Anxiety and Depression Scale and remission of depression as assessed by means of the Structured Clinical Interview for DSM IV Disorders by independent, blinded assessors at treatment termination. Secondary outcomes refer to quality of life.
\end{abstract}

Discussion: We investigate the efficacy of short-term psychodynamic psychotherapy in acute care and we aim to identify predictors for acceptance and success of treatment.

Trial registration: ISRCTN96793588

Keywords: Breast cancer, Depression, Short-term psychodynamic psychotherapy, Personality, Helping alliance, Quality of life

\section{Background}

Breast cancer is associated with multiple losses (e.g. regarding body image, sexuality, social relationships), strains (e.g. pain, fatigue) and threat to life. Depressive disorders are the most frequent mental comorbidities. The combined prevalence of major, minor depression and dysthymia in cancer patients was estimated at $22 \%$ $[1,2]$. In clinical routine, however, depression often escapes medical attention [3,4]. Without adequate

\footnotetext{
*Correspondence: ruediger.zwerenz@unimedizin-mainz.de

${ }^{\dagger}$ Equal contributors

'Department for Psychosomatic Medicine and Psychotherapy, University Medical Center Mainz, Untere Zahlbacher Str. 8, 55131, Mainz, Germany Full list of author information is available at the end of the article
}

treatment, depressive disorders in medically ill lead to substantial decrements of quality of life [5], longer inpatient treatment, prolonged work disability and inadequate illness behavior (e.g. lack of compliance) and even higher mortality [6].

Recently, there has been positive - somewhat limited evidence for the effectiveness of pharmacological and psychotherapeutic treatments with randomized controlled trials (RCT's) for depressed cancer patients, e.g. of cognitive behavior and problem-solving therapy for recently diagnosed, mildly to moderately depressed patients and of supportive-expressive group therapy for patients with advanced disease. Studies often suffer from methodological problems, such as selected or small

\section{Biomed Central}


samples, unclear or missing randomization, no manualization or control on treatment adherence. Unfortunately, only a minority of the trials has adequately assessed depression. A recent study ascertained clear preferences of cancer patients regarding speaking about their concerns and fears rather than accepting psychopharmacological treatment [7].

There has been increasing evidence supporting individual supportive-expressive psychotherapy as an effective short-term psychodynamic psychotherapy (STPP) for various mental disorders such as depression [8], generalized anxiety [9] and social phobia [10]. Based on the experience of developing a German manual [11] and training therapists in a multicenter RCT by two authors (MEB, $\mathrm{AH})$, a specific treatment manual was developed for treating depression in breast cancer patients [12]. We assumed that psychodynamic treatment is suitable to deal with the intrapsychic and interpersonal conflicts generated by the experience of cancer. We also assumed that maladaptive interpersonal relationship patterns play a pivotal role in this context and that therapeutic changes of these patterns lead to remission or improvement of depression. Also, the combination of interpretative and supportive treatments renders supportive-expressive psychotherapy flexible to deal with crises in the course of a potentially lifethreatening disease.

There is still a lack of knowledge on predictors of outcome of psychotherapy. Blatt \& Zuroff [13] found that the success of short-term outpatient treatment depended mostly on two factors: the quality of the therapeutic relationship and patients' pretreatment personality. In particular, those patients who were perfectionistic or self-critical before treatment improved less than those with low perfectionism. They obviously found it difficult to relate to their therapists in the time-limited treatment of depression. Thus, an additional issue of our trial is to determine the effects of personality and quality of the therapeutic relationship on treatment outcome.

\section{Methods/design}

In a multicenter trial, patients are recruited in the centers of Mainz and Leipzig in close cooperation with gynecological and oncological centers in the respective regions (list of cooperating clinics cf. appendix). Assessments are done by independent, trained and supervised research-assistants, who are blind to the intervention. Quality assurance is performed by the independent Interdisciplinary Center for Clinical Trials with regular monitoring visits including source data verification of all randomized patients, control of patient existence and written consent of all screened patients. Monitoring is defined in a monitoring manual and all results of the monitoring are written down in monitor reports.

\section{Participants}

Members of the oncological teams report eligible consecutive patients to trained research assistants. Following detailed information those who provide written consent with study participation are entered into the study and fill out the screening questionnaire. Patients are required to fulfill the test criteria on the Hospital Anxiety and Depression Scale (HADS, depression score $\geq 8$ ) and a diagnosis of a depressive disorder by the Structured Clinical Interview for DSM IV Disorders (SCID-I). Patients are randomized to the intervention or control group only if a diagnosis of a depressive disorder is made based on the aforementioned criteria. Inclusion and exclusion criteria are listed in Table 1.

\section{Intervention}

The intervention group is offered a manualized STPP adapting the approach of Luborsky et al. $[8,14]$ to the specific needs of depressed breast cancer patients [12]. Following the concept of the Core Conflict Relationship Theme (CCRT), depression is conceptualized in the context of intrapsychic and interpersonal conflicts. The CCRT is the treatment focus characterizing a maladaptive relationship pattern consisting of a wish, the response of the other and of the self. Five pre-treatment sessions include history taking (also the basis for formal application to the health insurance) and eliciting relationship episodes in the relationship interview to formulate the CCRT. The treatment agreement is established in one of the sessions. The therapist shares the CCRT with the patient, informs him about depression and about the treatment rationale in order to engage him in treatment. In the initial treatment phase (sessions 1-6), the therapist encourages building a positive alliance and links depressive symptoms to the CCRT. In the middle phase (sessions 7 to 12), the therapist refines the CCRT based on further relationship episodes from past, ongoing relationships and the relationship to the therapist. The vulnerability to depression is reduced when its dynamic can be understood from different points of view. In the termination phase (13 to 18) the therapist focuses on the issues of separation and the patient's ability to transfer the tools acquired in treatment to daily life. Booster sessions $(19,20)$ help to consolidate treatment progress. Treatment principles explicitly specified consider both the depressed state (e.g. dealing with helplessness/ hopelessness, anger, suicidality, negative attribution style) and the chronic and life-threatening disease (e.g. realistic treatment goals, here and now perspective, resource orientation). Psychodynamic therapists are certified or advanced trainees with psycho-oncological experience. They are trained in the manualized treatment during workshops before starting treatments and are regularly supervised during the treatments. For the supervision, each patient 
Table 1 Inclusion and exclusion criteria

\begin{tabular}{ll}
\hline Inclusion criteria: & Diagnosis of breast cancer \\
$\cdot$ & Curative treatment \\
$\cdot$ & - German language $18-70$ \\
$\cdot$ & Depression score (HADS $\geq 8)$ \\
$\cdot$ & Depressive disorder according to SCID-I (ICD-10 diagnoses: depressive episode F32.-, recurrent depressive episode \\
& F33.-; Dysthymia F34.1, adjustment disorder F43.21) \\
$\cdot$ - Written consent with study participation & - Severe medical conditions (metastases, cognitive impairments) \\
Exclusion criteria: & disorders except for cluster C, organic mental disorder) \\
$\cdot$ Concurrent psychotherapeutic treatment
\end{tabular}

receiving the STPP intervention is presented at three time points (formulation of the CCRT after the relationship interview, in the middle phase of STPP and in the termination phase of STPP). Supervision is free of charge for the participating psychotherapists. Each session is videotaped. For the evaluation of treatment adherence and competence cf. assessment.

\section{Assessments}

At baseline, patients fill out the HADS [15] in the German version (HADS-D) [16], a standardized questionnaire devised for the assessment of depression and anxiety in somatic illness with 14 items. Quality of life is assessed by the generic 30-item questionnaire of the European Organization for Research and Treatment of Cancer Quality of Life Questionnaire - Core 30 (EORTC QLQ-C30) [17] differentiating a global health status, functioning (physical, role, emotional, cognitive, social) and symptoms (e.g. fatigue, nausea and vomiting, pain etc.). Furthermore, the breast cancer specific quality of life module (EORTC QLQ-BR23) [18] which consists of functional scales (body image, sexual functioning, sexual enjoyment, future perspective) and symptom scales (system therapy side effects, breast symptoms, arm symptoms and upset by hair loss) is used. The validated German version of the Depressive Experience Questionnaire (DEQ) [19] is used to identify prognostically relevant personality dimensions of dependency (loneliness, helplessness, fear of rejection), perfectionism or self-criticism (worthlessness, failure, guilt, critical selfmonitoring) and self-efficacy (ambitious, competitive and self-reliant stance). The Multidimensional Fatigue Inventory (MFI) [20] is a 20 item self-report instrument covering the dimensions general fatigue, physical fatigue, reduced activity, reduced motivation and mental fatigue. The German version [21] of the Helping Alliance Questionnaire (HAQ) [14] an 11-item rating scale for assessing perceptions of the therapeutic relationship and process is filled out by the patient and therapist at the end of psychotherapy. The Structured Clinical Interview for DSM-IV [22] is used for standardized ('gold standard') assessment of axis I diagnoses. The PACS-SE (Penn Adherence/Competence Scale) with 45 Items [23] is used in the German version [24] for independent assessment of therapist adherence and competence based on randomly selected videotaped therapy sessions.

\section{Objectives}

The main purpose of the ongoing trial is to determine the efficacy of the manualized STPP regarding remission of depression in breast cancer patients. Secondary outcomes refer to changes of quality of life and to the effect of subtype of depression on treatment outcome.

Based on non-responder analyses we intend to answer the question, which patients do not accept psychotherapeutic treatment for what reason and which characteristics (e.g. age, marital, social status, illness variables, type of depression) have an impact on treatment outcome?

\section{Hypotheses \\ Primary outcome:}

1) Higher rate of remission in STPP vs. 'treatment as usual' (TAU) at treatment termination.

\section{Secondary outcomes:}

2) Six months after the end of treatment lower rate of depression and a higher quality of life among the STPP group compared to TAU.

3) Better outcome in dependent/anaclitic vs. selfcritical/ perfectionistic depression (DEQ).

\section{Study design}

In the multicenter randomized prospective trial breast cancer patients with comorbid depression who fulfill 
inclusion criteria either get STPP or TAU. Figure 1 gives an overview of the study design, time-points of assessments measures used and projected numbers.

We plan to recruit $\mathrm{N}=90$ participants per group. To ensure adequate randomization stratified for the center, the random assignment to STPP or TAU was performed separately by research staff from the responsible center (not engaged in the project) using a computer-generated [25] number series of random length (contained within closed, opaque envelopes.).

STPP entails five pre-treatment and up to 20 therapeutic sessions over a period of six months. In each center a close collaboration was established with about 10 psychodynamic psychotherapists in private practice who

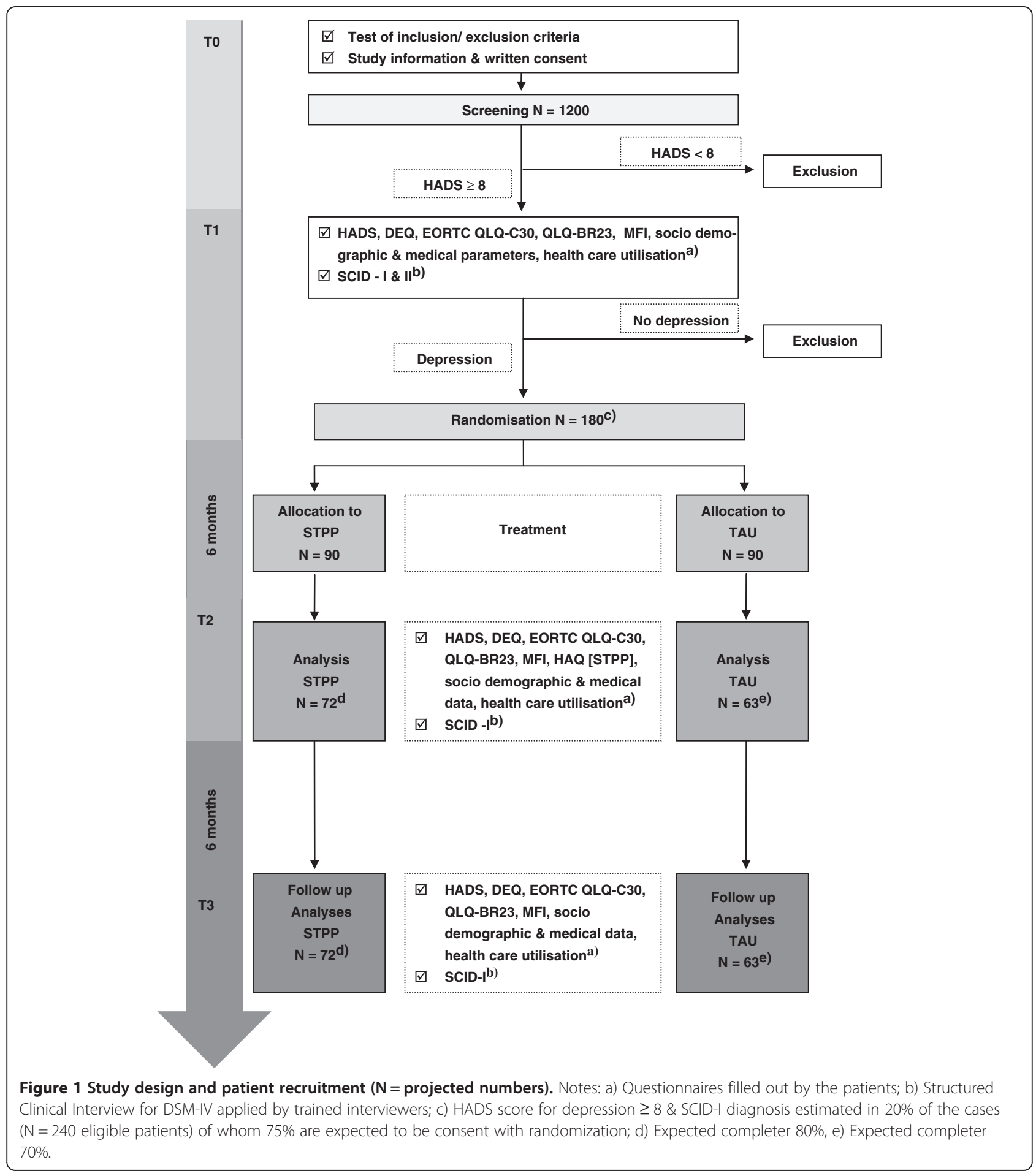


were trained according to the manual and supervised on a regular basis. Intensive training (at least $2 \times 2$ hours) is performed in a group format by the respective local study center prior to entering the first patient. Supervision is performed on a monthly basis (3 hours) in the group. In order to assure adherence to the manual, it is recommended that therapists present patients in supervision three times (initial formulation of CCRT based on relationship episode interview, middle and termination phase of treatment).

Patients randomized to the TAU condition are not offered STPP, but obtain written information on local cancer counseling centers and get written information on psychodiagnostic findings to their general practitioner who may then initiate antidepressant treatment ('as usual'). Utilization of medical, psychotherapeutic and psychopharmacological treatment is documented throughout the study.

\section{Outcome}

Remission of depression is defined by reduction of the HADS depression-score (at least by 2 points) and remission of depression (SCID-I-diagnosis) at treatment termination.

\section{Sample size calculation}

While planning this study no meaningful published data on remission rates for STPP vs. TAU for the treatment of depression in breast cancer were available. For STPP of depression remission rates between $45 \%$ and $70 \%$ at post-therapy assessment were published [26], while remission rates in primary-care settings (comparable to TAU) ranged between $20 \%$ and about 50\% (6 months) [27-29]. For patients with complex medical illness like cancer and co-morbid depressive disorders we expect lower remission rates for TAU and also for STPP. For this highly burdened population we pragmatically expected a spontaneous remission rate of $25 \%$ for TAU and 50\% for STPP in our sample size calculation.

In order to identify group differences of $25 \%$ (25\% remission in TAU vs. $50 \%$ in STPP) as based on $\chi^{2}$-Test (two-tailed) and a power of 0.80 a total sample of $\mathrm{N}=156$ is required, taking into account a slightly higher dropout rate of $30 \%$ in the TAU group vs. a $20 \%$ in STPP.

\section{Statistical analyses}

The primary endpoint remission after the treatment phase will be analyzed by a logistic regression with fixed effects for treatment and center and the baseline HADS value as covariate. The primary population for analysis will be the population intended to treat (ITT) consisting of all randomized patients. Dropouts will be regarded as non-remitters. For sensitivity the analysis will be repeated for the completers of treatment population. Additionally, the single components (HADS improvement of $\geq 2$ points, remission according to SCID) will be analyzed separately by the same analysis model as the combined endpoint.

Secondary analyses include the remissions at the end of the follow-up. Remissions at the end of the follow-up will be evaluated accordingly. The quality of life (QoL) questionnaires will be analyzed by an analysis of covariance (ANCOVA) model with fixed effects for treatment and center and the baseline values (HADS depression score \& Qol) as covariates. It is expected that the QoL questionnaires will not be linear across the study. Therefore QoL questionnaires after the follow-up will be evaluated by a linear model with fixed effects for treatment, center and time. There will be an indicator variable for the post treatment visit. The HADS value at baseline will serve as a covariate. For QoL data the observed values will be analyzed. However, if the number of missing data is more than $10 \%$, the data will be evaluated after multiple imputation of the data by a Markov chain Monte Carlo (MCMC) method. The impact of moderator variables (age, marital, social status, illness variables, type of depression) is tested in regression analyses.

\section{Safety aspects and medical complications}

Safety parameters will comprise newly occurring mental diagnoses and all serious adverse events that are reported during and up to six months after treatment.

The recording of adverse events will be restricted to psychological conditions. Formally, they are defined as any disorder classified by the International Classification of Diseases F00 - F99. A serious adverse event (SAE) is an adverse event that may occur at any time of the treatment phase or up to 6 months after end of treatment: results in death; is life-threatening; requires subject hospitalization or prolongation of existing hospitalization; results in persistent or significant disability/incapacity; is a congenital anomaly/birth defect.

Any SAE (according to the study specific definition) reported by the subject or detected by the local investigator will be collected during the trial and must be documented in case report forms. ICD-10 will be used by the local investigator to code the event. The clinical course of the SAE will be followed until it has changed to a stable condition or until end of follow-up phase, whatever comes first. In case of SAEs the Ethics Committee will be informed within 24 hours after the SAE becomes known.

\section{Ethical issues}

The final study protocol and the final version of the written informed consent form were approved by the Ethics Committee of the Federal State of Rhineland- 
Palatinate in Germany [reference number 837.380.06 (5478)] and the Ethics Committee of the University of Leipzig [reference number 218-2007]. The procedure set out in this protocol, pertaining to the conduct, evaluation, and documentation of this trial, were designed to ensure that all persons involved in the trial abide by Good Clinical Practice and the ethical principles described in the current revision of the Declaration of Helsinki [30]. The trial will be carried out in keeping with local legal and regulatory requirements. Before being admitted to the clinical trial, patients must consent to participate after the nature, scope, and possible consequences of the clinical trial have been explained in a form understandable to them. The patients must give written informed consent to participate in the study including their consent to publish.

\section{Discussion}

This is the first trial to determine the efficacy of a manualized STPP regarding remission of depression in breast cancer patients. Secondary outcomes refer to changes of quality of life and to the effect of subtype of depression on treatment outcome. Unlike previous studies we required a diagnosis of depression for study entry and we used remission of depression as a clinically relevant outcome criterion.

As we wish to contribute to evidence-based psychooncological care we chose as a control condition treatment as usual. We are aware that quality of care for the individual patient in the control condition may vary. However, we actually perform an augmented TAU condition by referring patients to a collaborating cancer counseling center which may provide individual counseling or further referral. We also take great care to send written and detailed findings on the comorbid depression diagnosis to the general practitioner of all the patients (IG and CG) who have given their written consent to do so. We make sure that we carefully assess the actual health care utilization by all patients during both follow-ups.

As we planned to provide an intervention for acute care, we recruit patients who are still in active treatment (chemotherapy, radiotherapy). Recruiting in the major local clinics we make sure that we could assess and contact the breast cancer patients with comorbid depression. We are aware, however, that a substantial proportion of patients would refuse study participation feeling strongly burdened by ongoing treatment. Based on non-responder analyses we intend to answer the question, which patients do not accept psychotherapeutic treatment for what reason [31]. Including the important dimensions of therapeutic alliance and personality dimensions shaping the experience and expression of depression we also ascertain who benefits most from treatment.

Previous studies of STPP adapting the approach of Luborsky et al. $[8,14]$ found no sex differences regarding treatment response [9]. Supportive-expressive psychotherapy has proven a robust model of short-term treatment for a diverse range of mental disorders. We therefore anticipate that it will be possible to transfer the treatment manual also to other kinds of cancer with comorbid depression less frequently studied - particularly in men.

\section{Abbreviations}

CCRT: Core Conflict Relationship Theme; DEQ: Depressive Experience Questionnaire; DSM-IV: Diagnostic and Statistical Manual of Mental Disorders (4 ${ }^{\text {th }}$ revision); EORTC QLQ-C30: European Organization for Research and Treatment of Cancer Quality of Life Questionnaire - Core 30; HADS: Hospital Anxiety and Depression Scale; HAQ: Helping Alliance Questionnaire (patient-, therapist-form); ICD-10: International Classification of Diseases (10 ${ }^{\text {th }}$ revision); ITT: Intention to treat; MFI: Multidimensional Fatigue Inventory; PACS-SE: Penn Adherence/Competence Scale; EORTC QLQ-BR23: Breast cancer specific quality of life module; QoL: Quality of life; RCT: Randomized controlled trials; SAE: Serious adverse event; SCID I: II, Structured Clinical Interview according to DSM-IV; STPP: Short-Term Psychodynamic Psychotherapy; TAU: Treatment as usual.

\section{Competing interests}

The authors declare that they have no competing interests.

\section{Authors' contributions}

The study design and assessments were conceptualized and developed by MEB, RS, AH, RZ, BHI, YB, SK, EB. Implementation and conduction of the study was coordinated by BHI, RZ, YB, SK and KL. RZ and MEB wrote an outline of the paper, which was carefully revised, edited and discussed by $J W, C R, K L, Y B, G W$ and EB. All authors read and approved the final manuscript.

\section{Acknowledgment}

We are grateful to all our participating patients without whom this study would not be possible. Furthermore we would like to thank the participating therapists and supervisors for conducting the treatments, our student research assistants for their important support in our study and last but not least our cooperating clinics and counseling centers (listed below in alphabetical order for the director of the institution) for their support in patient recruitment: Prof. Dr. A. du Bois \& Prof. Dr. C. Wulff, Dr. Horst Schmidt Clinic (HSK) Wiesbaden, Breast Center; Dr. S. Briest, University Medical Center Leipzig, Breast Center; Dr. V. Heyl, Asklepios Paulinen Clinic Wiesbaden, Breast Center; Prof. Dr. G. Hoffmann, St. Josefs-Hospital Wiesbaden, Breast Center; Dr. K. Josten \& Dr. O. Klein Medical office for Hematology and Oncology, Wiesbaden; Prof. Dr. U. Köhler, St. Georg-Hospital Leipzig, Breast Center; Prof. Dr. H. Kölbl, PD Dr. A. Lebrecht \& PD Dr. M. Schmidt, University Medical Center Mainz, Clinic for Gynecology; Prof. Dr. R.-D. Kortmann, University Medical Center Leipzig, Clinic for Radiotherapy; Dr. D. Langanke, St. ElisabethHospital Leipzig, Breast Center; Dipl.-Psych. Antje Lehmann-Laue,

Psychosocial Counseling Center for Cancer patients and relatives Leipzig; Prof. Dr. H. Madjar, German Diagnostic Clinic (DKD) Wiesbaden, Clinic for Gynecology; Prof. Dr. F.-J. Prott, Medical office for Radiotherapy Wiesbaden; Prof. Dr. H. Schmidberger, University Medical Center Mainz, Clinic for Radiotherapy; Dr. B. Stubert, HELIOS Clinic Schkeuditz, Breast Center; Dr. A. Werner, Tumor Center of Rhineland-Palatinate, Mainz; Prof. Dr. W. Wiest \& Dr. Ch. Hack, Clinic St. Vincenz Mainz, Breast Center. The study is funded by the German Cancer Aid (program 'Psychosocial Oncology' 01.10.2007 31.12.2012) with the reference numbers 107457 / 109379 (Mainz) and 107870 / 109381 (Leipzig).

\section{Author details}

'Department for Psychosomatic Medicine and Psychotherapy, University Medical Center Mainz, Untere Zahlbacher Str. 8, 55131, Mainz, Germany. 
${ }^{2}$ Interdisciplinary Center for Clinical Trials, University Medical Center Mainz, Langenbeckstr., 2, 55131, Mainz, Germany. ${ }^{3}$ Clinic for Radiotherapy, University Medical Center Mainz, Langenbeckstr., 2, 55131, Mainz, Germany. ${ }^{4}$ St. Josefs-Hospital, Breast Center, Beethovenstr., 20, 65189, Wiesbaden, Germany. ${ }^{5}$ Clinic for Gynecology, Breast Center, University Medical Center Mainz, Langenbeckstr., 2, 55131, Mainz, Germany. ${ }^{6}$ St. Georg-Hospital, Breast Center, Delitzscher Str. 141, 04129, Leipzig, Germany. ${ }^{7}$ St. Elisabeth Hospital, Breast Center, Biedermannstr., 84, 04277, Leipzig, Germany. ${ }^{8}$ Clinic for radiotherapy, University Medical Center Leipzig, Stephanstr., 9, 04103, Leipzig, Germany. ${ }^{9}$ Department for Medical Psychology and Medical Sociology, University Leipzig, Philipp-Rosenthal-Str. 55, 04103, Leipzig, Germany.

Received: 21 September 2012 Accepted: 19 November 2012 Published: 5 December 2012

\section{References}

1. Mitchell A, Chan M, Bhatti H, Halton M, Grassi L, Johansen C, Meader N: Prevalence of depression, anxiety, and adjustment disorder in oncological, haematological, and palliative-care settings: a meta-analysis of 94 interview-based studies. Lancet Oncol 2011, 12:160-174.

2. $\mathrm{Li}$ M, Fitzgerald P, Rodin G: Evidence-based treatment of depression in patients with cancer. J Clin Oncol 2012, 30:1187-1196.

3. Sharpe M, Strong V, Allen K, Rush R, Postma K, Tulloh A, Maguire P, House A, Ramirez A, Cull A: Major depression in outpatients attending a regional cancer centre: screening and unmet treatment needs. Br J Cancer 2004, 90:314-320.

4. Keller M, Sommerfeldt S, Fischer C, Knight L, Riesbeck M, Lowe B, Herfarth C, Lehnert T: Recognition of distress and psychiatric morbidity in cancer patients: a multi-method approach. Ann Oncol 2004, 15:1243-1249.

5. Jim HS, Small BJ, Minton S, Andrykowski M, Jacobsen PB: History of major depressive disorder prospectively predicts worse quality of life in women with breast cancer. Ann Behav Med 2012, 43:402-408.

6. Satin JR, Linden W, Phillips MJ: Depression as a predictor of disease progression and mortality in cancer patients: a meta-analysis. Cancer 2009, 115:5349-5361.

7. Hodges L, Butcher I, Kleiboer A, McHugh G, Murray G, Walker J, Wilson R, Sharpe M: Patient and general practitioner preferences for the treatment of depression in patients with cancer: how, who, and where? J Psychosom Res 2009, 67:399-402.

8. Luborsky L, Diguer L, Cacciola J, Barber J, Moras K, Schmidt K, DeRubeis R: Factors in outcome of short-term dynamic psychotherapy for chronic vs nonchronic major depression. J Psychother Pract Res 1996, 5:152-159.

9. Leichsenring F, Salzer S, Jaeger U, Kächele H, Kreische R, Leweke F, Ruger U, Winkelbach C, Leibing E: Short-term psychodynamic psychotherapy and cognitive-behavioral therapy in generalized anxiety disorder: a randomized, controlled trial. Am J Psychiatry 2009, 166:875-881.

10. Leichsenring F, Hoyer J, Beutel M, Herpertz S, Hiller W, Irle E, Joraschky P, König HH, de Liz TM, Nolting B, et al: The social phobia psychotherapy research network. The first multicenter randomized controlled trial of psychotherapy for social phobia: rationale, methods and patient characteristics. Psychother Psychosom 2009, 78:35-41.

11. Leichsenring F, Beutel M, Leibing E: Psychodynamic psychotherapy for social phobia: a treatment manual based on supportive-expressive therapy. Bull Menninger Clin 2007, 71:56-83.

12. Haselbacher A, Barthel Y, Brähler E, Imruck B, Kuhnt S, Zwerenz R, Beutel ME: Psychoanalytisch-orientierte fokaltherapie der depression bei Krebskranken. Psychotherapeut 2010, 55:321-328.

13. Blatt SJ, Zuroff DC: Empirical evaluation of the assumptions in identifying evidence based treatments in mental health. Clin Psychol Rev 2005, 25:459-486

14. Luborsky L, McLellan AT, Woody GE, O'Brien CP, Auerbach A: Therapist success and its determinants. Arch Gen Psychiatry 1985, 42:602-611.

15. Zigmond A, Snaith R: The hospital anxiety and depression scale. Acta Psychiatr Scand 1983, 67:361-370.

16. Hermann C, Buss U, Snaith R: HADS-D - Hospital Anxiety and Depression Scale Deutsche Version: Ein Fragebogen zur Erfassung von Angst und Depressivität in der somatischen Medizin, Testdokumentation und Handanweisung. Bern: Huber; 1995.

17. Aaronson NK, Ahmedzai S, Bergman B, Bullinger M, Cull A, Duez NJ, Filiberti $A$, Flechtner $\mathrm{H}$, Fleishman $\mathrm{SB}$, de Haes JC, et al: The european organization for research and treatment of cancer QLQ-C30: a quality-of-life instrument for use in international clinical trials in oncology. $\mathrm{J} \mathrm{Natl}$ Cancer Inst 1993, 85:365-376.

18. Sprangers MA, Groenvold M, Arraras JI, Franklin J, te Velde A, Muller M Franzini L, Williams A, de Haes HC, Hopwood P, et al: The European organization for research and treatment of cancer breast cancer-specific quality-of-life questionnaire module: first results from a three-country field study. J Clin Oncol 1996, 14:2756-2768.

19. Beutel M, Wiltink J, Hafner $C$, Reiner I, Bleichner F, Blatt S: Abhängigkeit und selbstkritik als psychologische dimensionen der depression - validierung der deutschsprachigen version des depressive experience questionnaire (DEQ). Z KI Psych Psychiatr 2004, 52:1-14.

20. Smets EM, Garssen B, Bonke B, De Haes JC: The multidimensional fatigue inventory (MFI) psychometric qualities of an instrument to assess fatigue. J Psychosom Res 1995, 39:315-325.

21. Bassler M, Potratz B, Krauthauser H: Der "Helping Alliance Questionnaire" (HAQ) von Luborsky. Psychotherapeut 1995, 40:23-32.

22. Wittchen H, Zaudig M, Fydrich T: SKID I - Strukturiertes Klinisches Interview für DSM-IV. Göttingen: Hogrefe; 1997

23. Barber J, Critis-Christoph P: Development of a therapist adherence/ competence rating scale for supportive-expressive dynamic psychotherapy: a preliminary report. Psychother Res 1996, 6:81-94.

24. Wiltink J, Edinger J, Haselbacher A, Imruck B, Beutel M: Adherence und competence in der psychotherapieforschung: neuere entwicklungen. Klin Diagn E 2010, 3:76-93.

25. Research Randomizer (Version 3.0). http://www.randomizer.org.

26. Leichsenring F: Comparative effects of short-term psychodynamic psychotherapy and cognitive-behavioral therapy in depression: a meta-analytic approach. Clin Psychol Rev 2001, 21:401-419.

27. Posternak MA, Solomon DA, Leon AC, Mueller TI, Shea MT, Endicott J, Keller MB: The naturalistic course of unipolar major depression in the absence of somatic therapy. J Nerv Ment Dis 2006, 194:324-329.

28. Posternak MA, Miller I: Untreated short-term course of major depression: a meta-analysis of outcomes from studies using wait-list control groups. $J$ Affect Disord 2001, 66:139-146.

29. Whiteford H, Harris M, McKeon G, Baxter A, Pennell C, Barendregt J, Wang J: Estimating remission from untreated major depression: a systematic review and meta-analysis. Psychol Med 2012, doi:10.1017/ S0033291712001717.

30. World Medical Association Declaration of Helsinki: Recommendations guiding physicians in biomedical research involving human subjects. JAMA 1997, 277:925-926

31. Applebaum AJ, Lichtenthal WG, Pessin HA, Radomski JN, Simay Gokbayrak N, Katz AM, Rosenfeld B, Breitbart W: Factors associated with attrition from a randomized controlled trial of meaning-centered group psychotherapy for patients with advanced cancer. Psychooncology 2011, doi:10.1002/ pon.2013.

doi:10.1186/1471-2407-12-578

Cite this article as: Zwerenz et al: Efficacy of psychodynamic short-term psychotherapy for depressed breast cancer patients: study protocol for a randomized controlled trial. BMC Cancer 2012 12:578.

\section{Submit your next manuscript to BioMed Central and take full advantage of:}

- Convenient online submission

- Thorough peer review

- No space constraints or color figure charges

- Immediate publication on acceptance

- Inclusion in PubMed, CAS, Scopus and Google Scholar

- Research which is freely available for redistribution 